\section{Evaluating an impression cytology grading system (IC score) in patients with dry eye syndrome}

E-M Haller-Schober ${ }^{1}$, G Schwantzer ${ }^{2}$, A Berghold ${ }^{2}$, M Fischl' ${ }^{1}$, A Theisl $^{1}$ and J Horwath-Winter

Keywords: impression cytology; grading system;

dry eye syndromes

Purpose To evaluate the IC score, a newly developed grading system for the morphological alterations of the ocular surface as a diagnostic tool in relation to type and severity of dry eye disease.

Methods Impression cytological specimens of 309 patients with simple dry eye (S-DE), 50 patients with Sjögren's syndrome and dry eye (SS-DE), and 39 patients with connective tissue disease and dry eye (CTD-DE) were examined. In all, 10 different morphological parameters were judged with points (IC subscores) and summarized to the IC score. IC score and subscores were correlated to tear function test results and with each other.

Results SS-DE patients had the highest IC scores and IC subscores. IC scores and IC subscores correlated significantly with the fluorescein staining of the cornea in all three patient groups. They were correlated to the rose bengal score in SS-DE and S-DE patients. The analysis of the IC subscores showed that there are significant correlations between most of the morphological alterations investigated. This underlines the importance of each parameter for the summarized IC score.

Conclusion The IC score provides detailed information about the morphological alterations of the bulbar conjunctiva. It has proven to be useful for the differentiation of dry eye diseases and might help in the investigation of the pathogenesis of these diseases as well as for follow-up and therapy control. Eye (2006) 20, 927-933. doi:10.1038/sj.eye.6702058; published online 12 August 2005

\section{Introduction}

The diagnosis of dry eye, a disorder of the ocular surface, is commonly based on typical symptoms, and various clinical tests evaluating tear film stability, tear secretion, and integrity of the cornea and conjunctiva by vital staining with fluorescein and rose bengal. Additionally, the morphology of the ocular surface can be investigated by conjunctival impression cytology. As the origin of ocular surface diseases is multifactorial, taking together the results of ocular surface and tear function tests provides a more complete understanding of this complex dynamic process.

Impression cytology is a simple, noninvasive technique that allows the collection of the superficial layers of the conjunctival epithelium for the morphological examination of the ocular surface. It was first described by Larmande and the ocular surface in 1954. Egbert et $a l^{2}$ documented the use of impression cytology for examining ocular surface disorders and goblet cell densities in 1977. This technique causes no discomfort for the patient, is repeatable, and offers the possibility of following the course of morphological alterations of the ocular surface.

The normal ocular surface (cornea and conjunctiva) is composed of a stratified nonkeratinizing epithelium, and in case of conjunctiva, different amounts of goblet cells, which produce the mucous part of the tear film. Pathological changes, such as squamous metaplasia of the epithelial cells, keratinization, Timsit ${ }^{1}$ for diagnosis of squamous neoplasia of
${ }^{1}$ Department of Ophthalmology, Medical University Graz, Graz, Styria, Austria

${ }^{2}$ Institute for Medical Informatics, Statistics, and Documentation, Medical University Graz, Graz, Styria, Austria

Correspondence: E-M Haller-Schober, Department of Ophthalmology, Medical University Graz,

Auenbruggerplatz 4, Graz, Styria A 8036, Austria

Tel: + 43316385 3187; Fax: +43316 385 7759/ 3261.

E-mail: eva.haller@ meduni-graz.at

Received: 28 February 2005 Accepted in revised form: 1 July 2005 Published online: 12 August 2005

Proprietary Interest: None 
and reduction of goblet cell density up to total goblet cell loss, occur in numerous disorders such as dry eye, Sjögren's syndrome, blepharokonjunctivitis, inflammatory keratokonjunctivitis, and vitamin A deficiency. ${ }^{3-12}$

For staging of the morphological changes of the conjunctiva, several grading systems have been developed. The first grading system published by Nelson $e t a l^{3}$ was based on the morphological appearance of the conjunctival epithelial and goblet cells, which was completed in $1988^{4}$ by the so-called mean individual epithelial cell area, according to the degree of squamous metaplasia, and the number of goblet cells. This grading system was scaled from 0 to 3 , based on the morphology of the epithelial cells, their staining behaviour, the nucleoplasmic ratio, as well as the density and the PAS staining of the goblet cells. This classification system is still widely used. Tseng ${ }^{5}$ published a grading system in 1985, scaled from 0 to 5 , which was based on the criteria described above, and the degree of keratinization of the epithelial cells in correlation to the three major steps of squamous metaplasia - loss of goblet cells, increase of cellular stratification, and keratinization. Adams $e a^{13}$ developed a grading system in 1988, scaled from 0 to 3 , taking into consideration the criteria of Tseng together with the quality of the cell-to-cell cohesion, that is, confluent or not. Heiligenhaus $e t \mathrm{al}^{14}$ added one morphological parameter to the staging of Tseng, the condensation of the nuclear chromatin. In the abovementioned grading schemes, ${ }^{3-5,13,14}$ the alteration of the epithelial cells and nuclei, goblet cell density, and morphological changes of goblet cells, as well as other morphological qualities were judged conjointly.

As we observed that the morphological changes of the bulbar conjunctiva, seen in ocular surface disorders, do not always occur in typical combinations, a new grading system (IC score) was developed. It includes more morphological parameters (IC subscores) to provide a complete picture of the conjunctival alterations. The parameters were separately judged and summarized to a total score (IC score), as already carried out by Aragona and co-workers on specimens from contact lens wearers. ${ }^{15}$ The IC score was designed for observing the course of dry eye patients over a long period, and for the statistical evaluation of morphological changes. ${ }^{16,17}$

The aim of this study was to evaluate the IC score on impression cytological specimens of 398 patients with dry eye syndrome as a diagnostic tool in relation to the type and severity of disease. The IC scores and the score points for each morphological parameter (IC subscores) of patients with simple dry eye (S-DE), patients with Sjögren's syndrome and dry eye (SS-DE), and patients with dry eye and connective tissue disease (CTD-DE) were calculated and compared.
The summarized IC scores and the IC subscores were correlated to the results of tear function tests such as tear film break up time (BUT), fluorescein staining of the corneal surface, Schirmer test without anaesthesia (Schirmer I), and rose bengal staining.

Finally, the IC subscores were statistically analysed to find out, if they correlate with the IC score as well as with each other, as proposed by authors, who graded the different morphological alterations conjointly.

\section{Patients and methods}

\section{Patients' data}

A computerized search of medical records from 1 January, 1994, to 31 July, 2003 identified all patients who presented at the outpatient clinic of the Department of Ophthalmology of the Medical University, Graz, Austria with ocular discomfort.

The study was designed in concordance with the declaration of Helsinki; patients' data were evaluated anonymously.

The ocular examination included tear film BUT, fluorescein staining of the corneal surface, Schirmer I, rose bengal staining, and impression cytology of the bulbar conjunctiva, as described previously. ${ }^{17}$ In brief, tear film BUT was measured by touching the inferotemporal bulbar conjunctiva with a fluorescein sodium strip, wetted with a preservative-free isotonic sodium chloride solution. The mean value of a total of three measurements was recorded. The corneal surface was examined under blue-light illumination with a biomicroscope after fluorescein instillation into the tear film. The intensity of fluorescein staining of the cornea was graded for each eye using a four-point scale (0, no staining; 1, less than one-third; 2 , less than two-thirds; and 3, more than two-thirds staining of the cornea).

A 5-min Schirmer I test without anaesthesia was performed on closed eyes using commercially available $5 \times 35$-mm paper strips (Clement Clarke International Ltd, London, UK). Rose bengal staining was performed by adding one drop (about $20 \mu \mathrm{l}$ ) of $1 \%$ rose bengal solution to the bulbar conjunctiva and the reaction was classified according to the van Bijsterveld scoring system. ${ }^{18}$ The results of both eyes were averaged.

The criteria for inclusion of patients were typical symptoms of dry eye such as dryness, burning, itching, foreign body sensation as well as ocular pain, and red or easily fatigued eyes, a reduced tear film BUT below $10 \mathrm{~s}$, and interpretable impression cytological specimens of the bulbar conjunctiva. A total of 538 patients met the inclusion criteria.

Contact lens wearers, patients with allergic conjunctivitis (with the presence of eosinophilic 
granulocytes in the inferior tarsal conjunctiva after Giemsa staining), patients with active infectious conjunctivitis, patients after a chemical injury or recent ocular surgery, patients with blepharospasm or with abnormal blinking as well as patients with superior limbal keratoconjunctivitis, and patients with ocular pemphigoid or with graft-versus-host disease (140 in total) were excluded.

The remaining 398 patients were divided into the following groups of dry eye disease:

(1) $S$-DE: patients with simple dry eye without an autoimmune disease: $n=309$ (77.6\%),

(2) $S S-D E$ : patients with primary or secondary Sjögren's syndrome who met the criteria based on the classification of Vitali et al: ${ }^{19} n=50(12.6 \%)$, and

(3) CTD-DE: patients with dry eye and connective tissue disease who did not match the criteria of Sjögren's syndrome such as rheumatoid arthritis, systemic lupus erythematosus, systemic sclerosis and others: $n=39(9.8 \%)$. For demographic and clinical data see Table 1 .

\section{Impression cytology}

Impression cytology specimens were obtained after topical anaesthesia with $0.4 \%$ oxybuprocaine from the superior and inferior bulbar conjunctiva (12 o'clock, 6 o'clock position) of both eyes of each patient. Cellulose acetate filter sheets (type VC, VCWP 04700, Millipore Corp., Bedford, MA, USA) with $0.10 \mu \mathrm{m}$ pores were cut into strips of about $3-10 \mathrm{~mm}$, and applied to the bulbar conjunctiva dull side down. With a flat, round-tipped forceps, the strips were gently pressed onto the ocular surface for a few seconds, and removed with a peeling motion. With this method, one to two epithelial cell sheets can be harvested. The filter strips were placed on slides on which double-sided adhesive tape had been applied, and air-dried. The specimens were stained with a combination of Gill's modified Papanicolaou stain and periodic acid-Schiff (PAS) reaction, as described by Tseng, ${ }^{5}$ placed in xylene to clear, and permanently mounted on a glass slide in Pertex ${ }^{\circledR}$ xylene-soluble mounting medium (medite, Germany), covered with a coverslip.

Examination under a light microscope (Zeiss, Axioscop) was always performed by the same researcher (HS-EM) without the knowledge of the clinical status. The following 10 morphological parameters were examined and judged with points (IC subscores) from 0 (normal) to 3 (highly pathological): quality of cell-to-cell adhesion (epithelial cell sheet confluent or not), degree of squamous metaplasia (nucleo/cytoplasmic correlation), tendency for keratinization, nuclear changes: type and frequency, as well as morphology and density of conjunctival goblet cells. According to the findings of Rivas et $a l,{ }^{20}$ the goblet cell densities were judged as normal (abundant), slightly, moderately, and distinctly reduced (single to no goblet cells). Morphology and density of superficial mucus, and the presence or absence of inflammatory cells were also judged. Thus, when summarized to the IC score, a maximum count of 30 points may be reached (Table 2, Figure 1a-f).

\section{Statistical analysis}

Data were entered into a computerized database and analysed. Continuous variables are reported as mean ( \pm standard deviation (SD)) and categorical data are displayed as frequencies with percentages in parentheses. To determine the statistical significance of group differences among the three study groups, the Kruskal-Wallis test was used for continuous data. Post hoc tests were performed with the Wilcoxon rank-sum test. Crosstabulations ( $\chi^{2}$ test, two-tailed Fisher's exact test) were used to assess the relationship between categorical variables. For correlations among continuous variables, we calculated the Spearman correlation coefficient.

All computations were carried out using the statistical package SPSS for Windows version $12.0 .1^{\circledR}$. A $P$-value of 0.05 or less was considered as statistically significant, except for post hoc tests where the significance level was corrected according to Bonferroni.

Table 1 Demographic data and clinical picture

\begin{tabular}{lccc}
\hline & $S-D E, \mathrm{n}=309$ & $S S-D E, \mathrm{n}=50$ & $C T D-D E, \mathrm{n}=39$ \\
\hline Age & $52.9( \pm 15.9)$ & $52.8( \pm 13.4)$ & $53.4( \pm 12.4)$ \\
Gender (female (\%)) & $235(76.1 \%)$ & $48(96.0 \%)^{*}$ & $35(89.7 \%)$ \\
Schirmer I (mm) & $10.8( \pm 9.5)$ & $1.4( \pm 1.7)^{*, \dagger}$ & $7.8( \pm 7.6)$ \\
BUT (s) & $5.0( \pm 2.4)$ & $2.0( \pm 1.8)^{*, \dagger}$ & $4.3( \pm 2.7)$ \\
Corneal staining score (0-3) & $0.4( \pm 0.7)$ & $1.5( \pm 1.0)^{*, \dagger}$ & $0.7( \pm 0.9)$ \\
Rose bengal staining score (0-9) & $2.6( \pm 2.4)$ & $7.5( \pm 1.8)^{*, \dagger}$ & $3.7( \pm 2.5)^{\ddagger}$ \\
\hline
\end{tabular}

Statistically significant differences between groups: *SS-DE and S-DE; †SS-DE and CTD-DE; ${ }^{\ddagger}$ CTD-DE and S-DE. 
Table 2 Morphological parameters and IC points included in the IC score grading system

\begin{tabular}{|c|c|c|c|c|c|}
\hline & IC subscores & 0 & 1 & 2 & 3 \\
\hline A & Epithelial cell sheet & Intact, dense & Slightly loosened & Loosened & Single cells \\
\hline \multirow[t]{2}{*}{ B } & Degree of squamous metaplasia $=$ & None & Low graded & Distinct & Massive \\
\hline & nucleocytoplasmic ratio (N/C) & $1: 1-1: 3$ & $1: 4-1: 6$ & $1: 6-1: 10$ & $>1: 10$ \\
\hline $\mathrm{C}$ & Degree of keratinization & None & Low graded & Distinct & Massive \\
\hline $\mathrm{D}$ & Nuclear changes frequency & None & Sporadic & Several & Abundant \\
\hline E & Nuclear changes type & None & $\begin{array}{l}\text { Low graded: pycnosis, } \\
\text { dissociation of chromatin, } \\
\text { chromatin condensation }\end{array}$ & $\begin{array}{l}1+\text { snake-like } \\
\text { chromatin }\end{array}$ & $\begin{array}{l}2+\text { nuclear } \\
\text { fragmentation }\end{array}$ \\
\hline $\mathrm{F}$ & Goblet cell density & Normal & Slightly reduced & Distinctly reduced & $\begin{array}{l}\text { Sporadic or no } \\
\text { goblet cells }\end{array}$ \\
\hline G & Goblet cell morphology & Normal & - & - & Pathological \\
\hline $\mathrm{H}$ & Mucus amount & Normal & No mucus & Medium & Abundant \\
\hline I & Mucus morphology & $\begin{array}{l}\text { Granular } \\
\text { plaques }\end{array}$ & $0+$ exudate & 0 or $1+$ filaments & 1 or $2+$ aggregation \\
\hline K & Inflammatory cells & None & Sporadic & Several & Abundant \\
\hline
\end{tabular}

\section{Results}

A total of 398 patients ( 318 women and 80 men) of a clinical population suffering from dry eye were retrospectively analysed.

SS-DE patients showed the most pathological tear film and ocular surface parameters compared to S-DE patients $(P<0.001$ for BUT, corneal staining score, and rose bengal staining) and CTD-DE patients $(P<0.001$ for BUT, corneal staining score, and rose bengal staining), respectively. In CTD-DE patients, a significantly higher, that is, more pathological rose bengal staining score was found compared to S-DE patients $(P=0.014)$ (Table 1$)$.

Concerning the clinical diagnoses, the most pathological - that is, highest - IC scores (mean $=13.4$; $\mathrm{SD}=5.4$ ) were found in SS-DE patients. S-DE patients showed a significantly less pathological picture (mean $=10.0 ; \mathrm{SD}=4.9 ; P<0.001$ ) as well as CTD-DE patients (mean $=10.1 ; \mathrm{SD}=4.6 ; P=0.002$ ) (Table 3 ).

The IC subscores for each morphological parameter showed that the following features differed significantly in SS-DE patients when compared to S-DE patients. The quality of cell-to-cell adhesion, the degree of squamous metaplasia, the type and frequency of nuclear changes, the morphology of goblet cells, the morphology of the superficial mucus, and the goblet cell density were more pathologic in SS-DE patients. Additionally, the IC subscores for the degree of squamous metaplasia, and the type and frequency of nuclear changes were significantly higher in SS-DE patients than those of CTD-DE patients. There was no statistically significant difference in the tendency towards keratinization, the goblet cell density, and mucus amount, between the three patients groups.

Fluorescein staining of the cornea of each patient group was significantly correlated to the respective IC scores $(r=0.29, r=0.58$, and $r=0.47$ for S-DE, SS-DE, and
CTD-DE patients, respectively). The rose bengal score was correlated to the IC score in S-DE and SS-DE patients ( $r=0.30, r=0.32$, respectively). Tear film BUT and Schirmer I test results did not correlate to the IC score in any of the three patients groups.

Concerning the correlations to clinical tear film and ocular surface parameters, the IC subscores, especially quality of cell-to-cell adhesion, degree of squamous metaplasia, nuclear changes, number of goblet cells, mucous aggregation, and presence or absence of inflammatory cells showed the same correlation pattern compared to the IC score. No correlation was observed with tendency towards keratinization, goblet cell morphology, and mucous amount (data not shown).

All IC subscores were well correlated with the IC score in the three patients groups. The analysis of the IC subscores revealed significant correlations between most of the morphological alterations investigated except for the morphology of the superficial mucus. This parameter was only correlated with the presence of inflammatory cells in S-DE and SS-DE patients (data not shown).

\section{Discussion}

Impression cytology has become the standard technique to study squamous metaplasia of the conjunctiva and goblet cell loss in ocular surface diseases. Various grading schemes were used, most of them being derived from the schemes proposed by Nelson et al, ${ }^{3}$ Nelson, ${ }^{4}$ or Tseng. ${ }^{5}$ Grading schemes provide a quick means of gross assessment of the morphological appearance of the ocular surface, especially the bulbar conjunctiva and are therefore useful for diagnosis and follow-up.

The IC score presented here provides an adoption of the grading scheme published by Aragona et al ${ }^{15}$ including the following 10 morphological parameters: the quality of cell-to-cell adhesion (epithelial cell sheet 

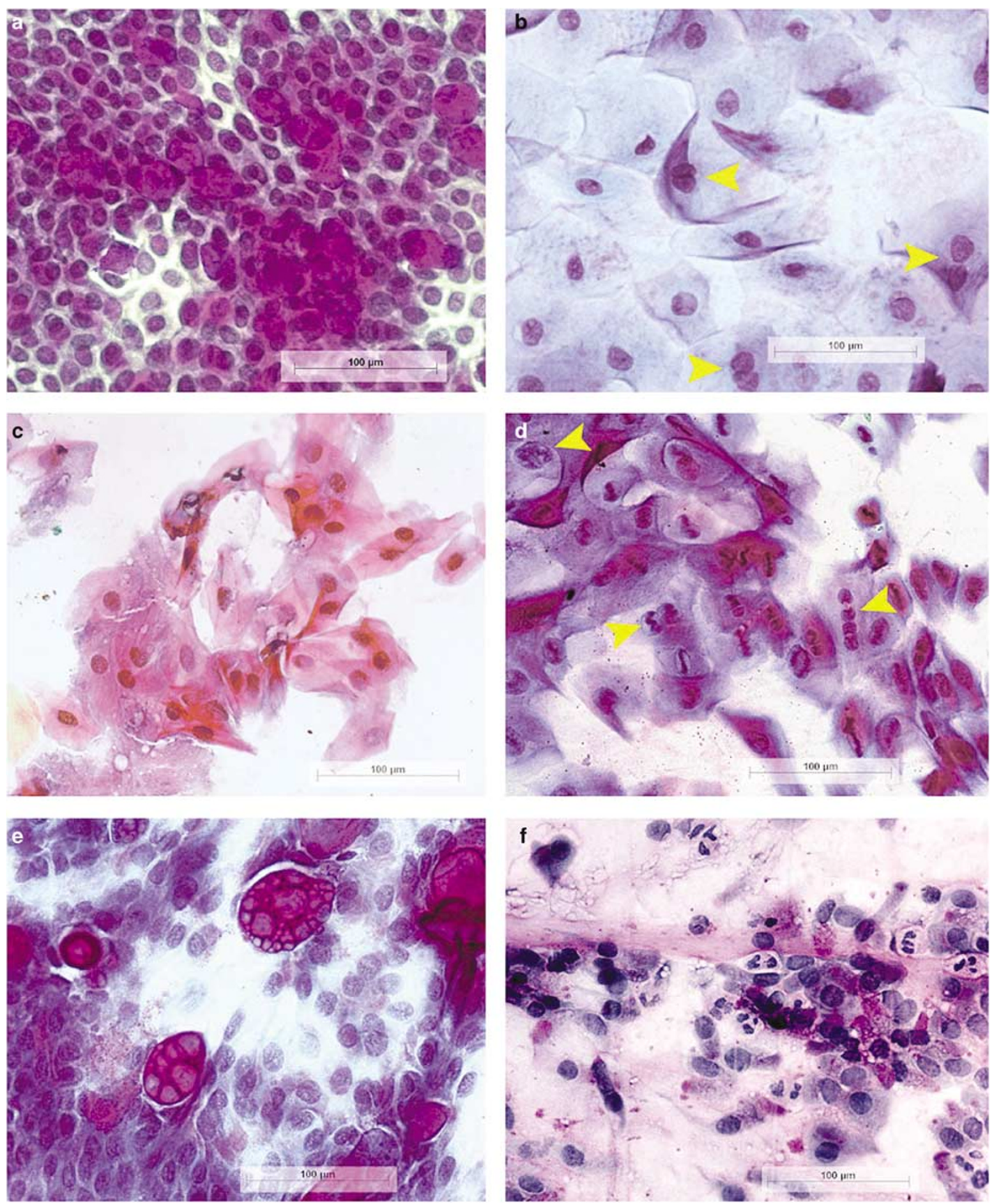

Figure 1 (a-f) Photomicrographs of impression cytology specimens, stained with PAS- and Gill's-modified Papanicolaou stain, microscopic magnification $\times 40$. (a) Normal cytological picture with round-shaped epithelial cells, dense staining round nuclei, and abundant goblet cells. (b) Distinct squamous metaplasia of the epithelial cells (high nucleocytoplasmic ratio) with double nuclei (arrowheads) and absence of goblet cells. (c) Distinct squamous metaplasia of the epithelial cells (high nucleocytoplasmic ratio) and different degrees of keratinization. (d) Distinct squamous metaplasia of the epithelial cells (high nucleocytoplasmic ratio) and different degrees of keratinization. All nuclei pathological altered: double nuclei, snake-like chromatin, and nuclear fragmentations (arrowheads). (e) Normal epithelial cells and pathological altered goblet cells. (f) Slight squamous metaplasia of the epithelial cells with normal nuclei. High mucous amount with mucous strains and polymorphonuclear leucocytes between the epithelial cells. Absence of goblet cells. 
Table 3 IC score of the study groups

\begin{tabular}{cccr}
\hline & $S-D E$ & $S S-D E$ & $C T D-D E$ \\
\hline IC score & $10.0( \pm 4.9)$ & $13.4( \pm 5.4)^{*, \dagger}$ & $10.1( \pm 4.7)$
\end{tabular}

Statistically significant differences between groups: *SS-DE and S-DE †SS-DE and CTD-DE.

confluent or not), the degree of squamous metaplasia (nucleocytoplasmic ratio), the tendency for keratinization, nuclear changes: type and frequency, the morphology and density of conjunctival goblet cells, as well as the type and amount of superficial mucus, and the presence or absence of inflammatory cells. As performed by Aragona et al, ${ }^{15}$ but different to the protocols of Nelson et al, ${ }^{3}$ Nelson, ${ }^{4}$ and Tseng, ${ }^{5}$ these parameters were judged individually resulting in the so-called IC subscores and summarized to the IC score. This provides the possibility to evaluate each morphological parameter separately, and to inter-relate each result, IC score or IC subscores, with diagnostic or clinical parameters as well. As previously carried out by Nepp et al, ${ }^{21}$ each IC subscore as well as the IC score may be combined to other morphological or clinical and tear film, and staining scores to obtain some kind of 'overall dry eye score'.

To compare the IC score and IC subscores with the results of diagnostic tests for dry eye disease, we examined patients of a clinical population, who were classified in three different groups: S-DE, SS-DE, and CTD-DE-patients, since they might have a higher risk for a severe course of disease.

As expected, SS-DE patients had the most pathological tear film and ocular surface parameters when compared to the other two groups, and CTD-DE patients showed a more pathological rose bengal staining score than S-DE patients. SS-DE patients presented the highest IC scores reflecting an intensely altered morphology of the ocular surface, as described by other authors, ${ }^{7,9,22,23}$ while S-DEor CTD-DE patients showed similar degrees of conjunctival alteration.

Comparing the IC subscores with the type of dry eye disease, these results were mainly confirmed, but the results for the tendency towards keratinization, the goblet cell density, and the mucus amount were not significantly different between the three patients groups. This might be due to the fact that loss of goblet cells and keratinization occur early after the onset of disease.

To the best of our knowledge most of the researchers investigated the inter-relations of the clinical tear film and ocular surface parameters with single morphological parameters such as degree of squamous metaplasia, goblet cell density, or nuclear changes, while in the study presented, these correlations were calculated for the IC subscores and the summarized IC score as well. A comparison of our results with previously postulated correlations is difficult, because the patients groups investigated were different over all the studies performed, and there were different localizations of specimen harvesting-12 o'clock, 6 o'clock position $v s$ many different parts of the conjunctiva including interpalpebral and palpebral regions.

In concordance to Pflugfelder, ${ }^{24}$ we found that in patients with SS-DE, the morphological alterations seen in impression cytology specimens correlated well with clinical findings, such as staining of the cornea and conjunctiva.

The IC subscores for the quality of the cell sheet, the degree of squamous metaplasia, the nuclear changes, the goblet cell density, mucous aggregation, and presence or absence of inflammatory cells were also correlated with tear film and ocular surface tests as described for the IC score. Our results are in concordance with Lee and co-workers, ${ }^{25}$ who found that the severity of squamous metaplasia correlated well with the intensity of the rose bengal score, and with Pflugfelder $e t a l^{8}{ }^{8}$ who described a correlation between goblet cell density and rose bengal staining. Bjerrum ${ }^{26}$ found a correlation between nuclear changes and rose bengal staining, which was also seen in our population. In our opinion, these findings demonstrate and underline that there is a close relationship between ocular surface staining and the IC subscores as well as the summarized IC score.

In our three patients groups, there was no correlation of the IC score and IC subscores with BUT, and Schirmer I test results. This is in contrast to the findings of Pflugfelder et $a l_{,}^{8}$ who observed a correlation of goblet cell density, and an inverse correlation between the degree of squamous metaplasia with Schirmer I test results in patients with ocular irritation.

As expected, all IC subscores were well correlated with the IC score in the three patients groups, and there were significant correlations between most of the morphological alterations investigated. The type and frequency of nuclear changes were moderately (CTD-DE patients) to strongly (S-DE and SS-DE patients) correlated, which demonstrates that nuclear changes such as snake-like chromatin or nuclear fragmentation reflect a highly pathological picture, independent of the frequency of these alterations. In concordance to Rivas and co-workers, ${ }^{10}$ we found no correlation between the type of nuclear changes, the degree of squamous metaplasia, and the presence of inflammatory cells in patients with SS-DE. Like Tseng, ${ }^{5}$ we found that a decreased goblet cell density was accompanied by an increasing nucleocytoplasmic ratio, but we did not see a distinct correlation of the inflammatory cell infiltrate with the presence of extensive squamous metaplasia in 
SS-DE patients, which was observed by Pflugfelder and co-workers. ${ }^{9}$

Overall, these results underline the important contribution of each morphological parameter investigated to the summarized IC score.

In summary, judging conjointly morphological alterations for grading the alterations of the (bulbar) conjunctiva, as carried out by several investigators before, gives a good, reproducible overview, but the IC score provides more detailed information, because each morphological parameter can be judged separately. For an overview, the resulting IC subscores are summarized to the IC score. In the study population presented here, the IC scores of patients with SS-DE are significantly different from the IC scores obtained from patients with S-DE or CTD-DE, thus providing a helpful diagnostic tool for the differentiation of these diseases.

As the specimens were always taken from the same positions, the comparison of the IC subscores obtained during an observation period might be helpful for the follow-up of the alteration of each morphological parameter.

Thus, the IC score provides an extensive evaluation of a lot of morphological alterations of the bulbar conjunctiva, and may therefore also serve as a valuable research tool for the investigation of the pathogenesis of dry eye disease as well as for therapy control.

\section{References}

1 Larmande A, Timsit E. L'intérêt de cyto-diagnostiques en ophtalmologie: communication préliminaire à propos $\mathrm{d}$ neuf cas de tumours du limbe scléro-cornéen. Bull Soc Ophthalmol Fr 1954; 19: 415-419.

2 Egbert PR, Lauber S, Maurice DM. A simple conjunctival biopsy. Am J Ophthalmol 1977; 84: 798-801.

3 Nelson JD, Havener VR, Cameron JD. Cellulose acetate impressions of the ocular surface. Dry eye states. Arch Ophthalmol 1983; 101: 1869-1872.

4 Nelson JD. Impression cytology. Cornea 1988; 7: 71-81.

5 Tseng CGT. Staging of conjunctival squamous metaplasia by impression cytology. Ophthalmology 1985; 92: 728-733.

6 Rolando M, Terragna F, Giordano G, Calabria G. Conjunctival surface damage distribution in keratoconjunctivitis sicca. An impression cytology study. Ophthalmologica 1990; 200: 170-176.

7 Petroutsos G, Paschides CA, Karakostas KX, Psilas K. Diagnostic tests for dry eye diseases in normal and dry eye patients with and without Sjögren's syndrome. Ophthalmic Res 1992; 24: 326-331.

8 Pflugfelder SC, Tseng SC, Yoshino K, Monroy D, Felix C, Reis BL. Correlation of goblet cell density and mucosal epithelial membrane mucin expression with rose bengal staining in patients with ocular irritation. Ophthalmology 1997; 104: 223-235.

9 Pflugfelder SC, Huang AJW, Feuer W, Chuchovski PT, Pereira IC, Tseng SCG. Conjunctival cytologic features of primary Sjögren's syndrome. Ophthalmology 1990; 97: 985-991.

10 Rivas L, Rodriguez JJ, Alvarez MI, Oroza MA, del Castillo JM. Correlation between impression cytology and tear function parameters in Sjögren's syndrome. Acta Ophthalmol (Copenhagen) 1993; 71: 353-359.

11 Rivas L, Oroza MA, Perez-Esteban A, Murube-del-Castillio J. Morphological changes in the ocular surface in dry eyes and other disorders by impression cytology. Graefe's Arch Clin Exp Ophthalmol 1992; 230: 329-334.

12 Natadisastra G, Wittpenn JR, West Jr KP, Muhilal RD, Sommer A. Impression cytology for detection of vitamin A deficiency. Arch Ophthalmol 1987; 105: 1224-1228.

13 Adams GGW, Dilly PN, Kirkness CM. Monitoring ocular disease by impression cytology. Eye 1988; 2: 506-516.

14 Heiligenhaus A, Koch JM, Kruse FE, Schwarz C, Waubke ThN. Diagnostik und Differenzierung von Benetzungsstörungen. Ophthalmologe 1995; 92: 6-11.

15 Aragona P, Ferreri G, Micali A, Puzzolo D. Morphological changes of the conjunctival epithelium in contact lens wearers evaluated by impression cytology. Eye 1998; 12: 461-466.

16 Haller-Schober E-M, Horwath-Winter J. Der IC-Score-Ein neuer Ansatz zur Interpretation von impressionszytologischen Präparaten. (IC-Score - a new approach to interpretation of impression cytological specimens). Spektrum Augenheilkd 2002; 16: 154-158.

17 Horwath-Winter J, Berghold A, Schmut O, Floegel I, Solhdju $\mathrm{V}$, Bodner $\mathrm{E}$ et al. Evaluation of the clinical course of dry eye syndrome. Arch Ophthalmol 2003; 121: 1364-1368.

18 Van Bijsterveld OP. Diagnostic tests in the sicca syndrome. Arch Ophthalmol 1969; 82: 10-14.

19 Vitali C, Bombardieri S, Moutsopoulos HM. Preliminary criteria for the classification of Sjögren's syndrome. Arthritis Rheum 1993; 36: 340-347.

20 Rivas L, Oroza MA, Perez-Esteban A, Murube-del-Castillo J. Topographical distribution of ocular surface cells by the use of impression cytology. Acta Ophthalmol 1991; 69: 371-376.

21 Nepp J, Abela C, Polzer I, Derbolav A, Wedrich A. Is there a correlation between the severity of diabetic retinopathy and keratoconjunctivitis sicca? Cornea 2000; 19: 487-491.

22 Rivas L, Alvarez MI, Rodriguez JJ, Murube J. Ophthalmological tests in patients with keratoconjunctivitis sicca with and without association of primary Sjogren's syndrome. Ger J Ophthalmol 1995; 4: 306-310.

23 Rivas L, Murube J, Shalaby O, Oroza MA, Sanz AI. Contribucion de la citologia de impresion al diagnostico differencial del sindome de Sjogren an la clinica oftalmologica. (Impression cytology contribution to differential diagnosis of Sjogren syndrome in the ophthalmological clinic). Arch Soc Esp Oftalmol 2002; 77: 63-72.

24 Pflugfelder SC. Tear fluid influence on the ocular surface. In: Sullivan DA, Dartt DA, Meneray MA (eds). Lacrimal Gland, Tear Film, and Dry Eye Syndromes 2. Plenum Press: New York, 1998, pp 611-617.

25 Lee SH, Tseng SCG. Rose bengal staining and cytologic characteristics associated with lipid tear deficiency. Am J Ophthalmol 1997; 124: 736-750.

26 Bjerrum KB. Snake-like chromatin in conjunctival cells of a population aged 30-60 years from Copenhagen city. Acta Ophthalmol Scand 1998; 76: 294-298. 